\title{
The use of a cycle ergometer to calculate myocardial perfusion reserve with $k-t$ sense-accelerated myocardial perfusion $M R$ imaging at 3.0 Tesla
}

\author{
Tim Lockie*1, Masaki Ishida1 ${ }^{1}$ Kapla de Silva1 ${ }^{1}$, Rupert Williams1, \\ Simon Redwood ${ }^{1}$, Sebastian Kozerke ${ }^{1}$, Michael Marber ${ }^{1}$, Eike Nagel ${ }^{1}$ and \\ Sven Plein ${ }^{2}$
}

\author{
Address: ${ }^{1}$ Rayne Institute, St Thomas Hospital, KCL, London, UK and ${ }^{2}$ Rayne Institute, St Thomas Hospital, KCL and Division of Cardiovascular \\ and Neuronal Remodelling, LIGHT Institute, University of Leeds, Leeds, UK \\ * Corresponding author
}

from 13th Annual SCMR Scientific Sessions

Phoenix, AZ, USA. 21-24 January 2010

Published: 21 January 2010

Journal of Cardiovascular Magnetic Resonance 2010, I2(SuppI I):P87 doi:I0.I 186/I532-429X-I2-SI-P87

This abstract is available from: http://jcmr-online.com/content/I2/SI/P87

(c) 2010 Lockie et al; licensee BioMed Central Ltd.

\section{Background}

Exercise induced stress testing is important to determine the physiological reserve of the myocardium. High heart rate (HR) and respiratory motion represent a challenge for $\mathrm{CMR}$ perfusion imaging requiring very fast data acquisition to minimise artefacts.

\section{Purpose}

To quantify perfusion at rest and exercise-induced stress in volunteers using $k$-space and time sensitivity encoding $(k$ $t$ SENSE) CMR perfusion.

\section{Methods}

\section{CMR}

$k$ - $t$ SENSE accelerated perfusion CMR was performed on a 3 T Philips Achieva ${ }^{\circledR}$ system using $0.025 \mathrm{mmol} / \mathrm{kg} / \mathrm{min}$ Gd-DO3A-butrol and the following pulse sequence: saturation recovery gradient echo, repetition time/echo time $3.0 \mathrm{~ms} / 1.0 \mathrm{~ms}$, flip angle $15^{\circ}, 5 \times k$ - $t$ SENSE acceleration, 11 interleaved training profiles, WET pre-pulse (angles $\left.120^{\circ}, 90^{\circ}, 180^{\circ}, 140^{\circ}\right)$; delay $100 \mathrm{~ms}$, spatial resolution $1.8 \times 1.8 \times 10 \mathrm{~mm}^{3}, 3$ slices at each RR interval, 40 dynamic images. For blood pool saturation correction MR images with diluted Gd-DO3A-butrol injection (0.0025 $\mathrm{mmol} / \mathrm{kg}$ ) were also acquired.

\section{Subjects}

5 volunteers (age 21-35 years) with no history of cardiac disease.

\section{Exercise protocol}

A specially adapted supine cycle ergometer was used and attached to the sliding table inside the CMR scanner such that the subject could exercise without leaving the table. A standardised exercise protocol was used with increments of $20 \mathrm{~W}$ each minute for 6 minutes at a rate of $60 \mathrm{rpm}$. Rate pressure product (RPP) calculated as peak systolic blood pressure $\times$ peak HR was recorded at peak exercise.

\section{Analysis}

The mid-slice was used in each subject for further analysis. After correcting saturation of the blood signal, arterial input and myocardial output time-intensity curves were analyzed with a Patlak plot method to quantify global myocardial $\mathrm{K} 1$ at rest and stress. Then, absolute global myocardial blood flow (MBF) at rest and stress and perfusion reserve were calculated from $\mathrm{K} 1$ by using extraction fraction of Gd contrast agent ${ }^{1-3}$

\section{Results}

All subjects successfully completed the exercise protocol. Resting MBF was $90.5 \pm 12.1 \mathrm{ml} / \mathrm{min} / 100 \mathrm{~g}$ compared to 
Table I: Full dataset of volunteers

\begin{tabular}{|c|c|c|c|c|c|c|}
\hline & $\mathbf{K I}$ & & & MBF & $(=K I / E F)$ & \\
\hline & & & & $E F=0.5^{*}$ & $\mathrm{EF}=0.3^{*}$ & \\
\hline Volunteer & Rest & Stress & Reserve(stress/rest) & rest & stress & MPR \\
\hline 1 & 47.9 & 182.2 & 3.8 & 95.8 & 607.3 & 6.3 \\
\hline 2 & 41.4 & 98.5 & 2.4 & 82.8 & 328.3 & 3.9 \\
\hline 3 & 38.4 & 64.3 & 1.7 & 76.8 & 214.3 & 2.8 \\
\hline 4 & 54 & 84.5 & 1.6 & 108 & 281.7 & 2.6 \\
\hline 5 & 44.6 & 74.7 & 1.7 & 89.2 & 249.0 & 2.8 \\
\hline mean & 45.3 & 100.8 & 2.2 & 90.5 & 336.1 & 3.6 \\
\hline SD & 6.0 & 47.1 & 0.9 & 12.1 & I57.3 & 1.5 \\
\hline
\end{tabular}

*Extraction fraction (EF) of 0.5 at rest and 0.3 stress forGd-DTPAbased on data acquired at $1.5 \mathrm{~T}$

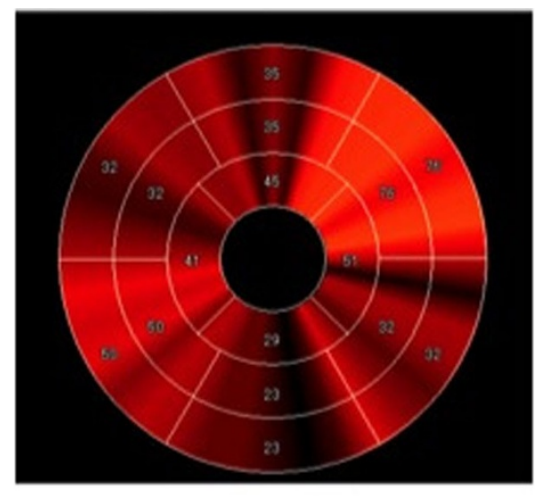

b)

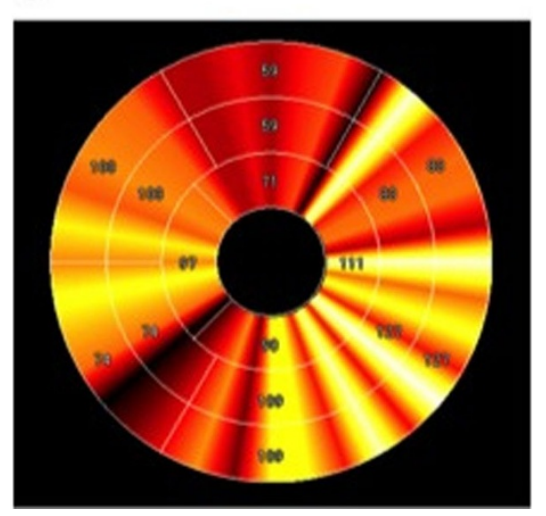

Figure I

Patiak plot for mid slice at a) rest and, b) stress showing globally increased KI measurements. stress of $336.1 \pm 157.3 \mathrm{ml} / \mathrm{min} / 100 \mathrm{~g}(\mathrm{P}<0.008)$, giving an MPR of $3.6 \pm 1.5$, consistent with physiological values. RPP was $17556 \pm 1209$ indicating equivalent workload across all subjects. See table 1 Figure 1.

\section{Conclusion}

$k$ - $t$ SENSE can be used to acquire perfusion CMR data during ergometer stress and to determine global myocardial perfusion reserve in healthy volunteers. More studies are required to determine regional flow distribution but this technique holds potential for the functional assessment of patients with cardiac disease. 